\title{
Comparação entre a correção cilíndrica total e o equivalente esférico na realização da perimetria computadorizada
}

\author{
Comparison between the full cylindrical correction and the spherical equivalent in the \\ execution of automated perimetry
}

\author{
Paulo Leonardi Filho ${ }^{1}$ \\ Cristiano Caixeta-Umbelino ${ }^{2}$ \\ Marcela Colussi Cypel-Gomes ${ }^{3}$ \\ Ricardo Nunes Eliezer ${ }^{4}$ \\ Renato King'leufus Pinheiro ${ }^{4}$ \\ Niro Kasahara ${ }^{5}$ \\ Maurício Della Paolera ${ }^{6}$
}

Santa Casa de Misericórdia de São Paulo - Departamento de Oftalmologia - Setor de Glaucoma. Rua Cesário Motta Junior, 112 - CEP 01221-020 - São Paulo (SP).

${ }^{1}$ Fellow do Setor de Retina e Vítreo do Departamento de Oftalmologia da Santa Casa de Misericórdia de São Paulo.

2 Fellow do Setor de Glaucoma do Departamento de Oftalmologia da Santa Casa de Misericórdia de São Paulo.

${ }^{3}$ Fellow do Setor de Urgências do Departamento de Oftalmologia da Santa Casa de Misericórdia de São Paulo e do Setor de Retina e Vítreo do Departamento de Oftalmologia da Universidade de São Paulo - USP.

${ }^{4}$ Médico Voluntário do Setor de Glaucoma do Departamento de Oftalmologia da Santa Casa de Misericórdia de São Paulo. Pós graduando, nível doutorado, pela Universidade de São Paulo - USP.

5 Médico Voluntário do Setor de Glaucoma do Departamento de Oftalmologia da Santa Casa de Misericórdia de São Paulo.

${ }^{6}$ Médico Assistente do Setor de Glaucoma do Departamento de Oftalmologia da Santa Casa de Misericórdia de São Paulo.

Endereço para correspondência: Cristiano Caixeta Umbelino, Rua Martinico Prado n 392 apt $^{\circ} 92$

CEP 01224-010 - São Paulo (SP)

E-mail: criscaixeta@ig.com.br

Recebido para publicação em 27.11.2002

Versão revisada recebida em 18.06.2003

Aprovação em 17.12.2003

Nota Editorial: Pela análise deste trabalho e por sua anuência na divulgação desta nota, agradecemos ao Dr. Ítalo Mundialino Marcon.

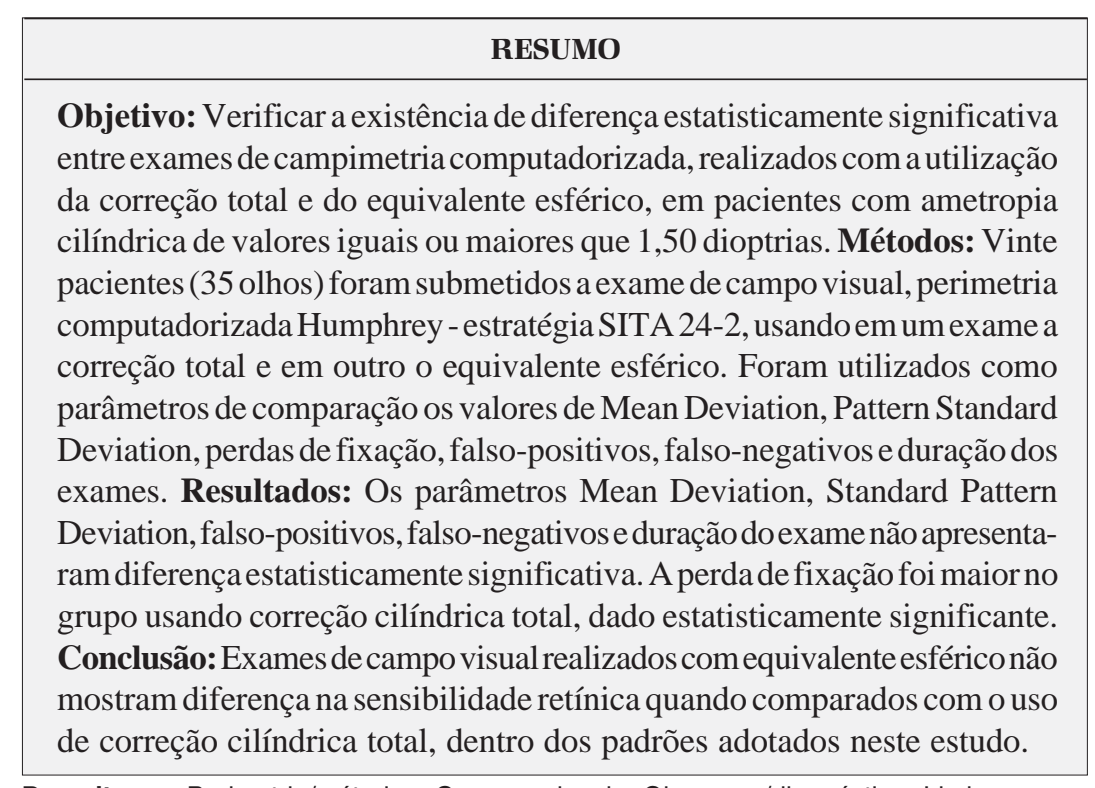

Descritores: Perimetria/métodos; Campos visuais; Glaucoma/diagnóstico; Limiar sensorial; Erros de refração/diagnóstico; Estudo comparativo

\section{INTRODUÇ̃̃̃O}

O exame do campo visual é essencial no diagnóstico e acompanhamento pacientes com glaucoma.

A campimetria computadorizada é um exame útil na prática clínica oftalmológica, incorporando avanços que aumentam a praticidade na realização do exame, bem como sua confiabilidade ${ }^{(1-2)}$. As principais vantagens da campimetria computadorizada são: teste do campo visual pelo método estático (diferentes intensidades luminosas em um mesmo ponto), ao invés do modo cinético, habitualmente utilizado na campimetria manual; redução da subjetividade do examinador; monitorização constante da fixação; capacidade de reteste automático de pontos anormais; múltiplas estratégias de teste, de acordo com a necessidade do examinador ${ }^{(1)}$.

Para a realização do exame de campimetria em pacientes com ametropia, deve-se utilizar a correção óptica que propicie a melhor acuidade visual, para que haja percepção retínica adequada dos estímulos luminosos emitidos durante o exame ${ }^{(1-3)}$

No exame de campimetria computadorizada, as ametropias cilíndricas até 0,25 dioptrias podem ser ignoradas ${ }^{(1)}$. Nas de valores 0,50 ou 0,75 dioptrias, 
faz-se o uso do equivalente esférico, e para as de valores iguais ou maiores do que 1,00 dioptria deve ser feito o uso da correção total.

Embora tal procedimento seja o preconizado, nenhum estudo científico foi realizado a fim de demonstrar as possíveis alterações na sensibilidade ao exame causadas pela utilização do equivalente esférico, ao invés da correção total.

O objetivo do estudo foi verificar se há diferença estatisticamente significativa entre a campimetria realizada com a correção óptica total e a com o equivalente esférico, em pacientes com astigmatismo de grau igual ou superior a 1,50 dioptrias cilíndricas, sem outra alteração oftalmológica que possa interferir na acuidade visual, através da análise da sensibilidade aos estímulos luminosos durante o exame.

\section{MÉTODOS}

O estudo foi realizado no Setor de Glaucoma do Departamento de Oftalmologia da Santa Casa de Misericórdia de São Paulo, no período de dezembro de 1999 a julho de 2000.

Os critérios de inclusão para o estudo foram: idade entre 16 e 60 anos, ausência de outra alteração oftalmológica que provoque diminuição da acuidade visual, acuidade visual com correção igual a 20/20, compreensão e cooperação do exame a ser realizado.

Os pacientes foram submetidos a exame oftalmológico completo, realizados pelo mesmo médico, sendo devidamente informados de que participavam de um estudo científico. Nos pacientes com idade inferior a 40 anos, foram realizados exames refratométricos estáticos sob cicloplegia (instilação de uma gota de ciclopentolato a $1 \%$ e uma gota de tropicamida a $1 \%$ com intervalo de 5 minutos).

Foram examinados 35 olhos de 20 pacientes (20 olhos direitos e 15 olhos esquerdos): a média dos valores dióptricos das ametropias cilíndricas foi de 2,05 dioptrias $( \pm 0,73)$ e valores extremos de 1,50 a 4,50 dioptrias. A média dos valores dióptricos das ametropias esféricas foi de $-2,50$ dioptrias $( \pm 3,90)$ e valores extremos variando de $-12,75 \mathrm{a}+7,00$ dioptrias.

A média da idade dos pacientes foi de 24,5 anos $( \pm 8,02)$ e os valores extremos variando de 16 a 51 anos, sendo 11 pacientes do sexo masculino e 9 pacientes do sexo feminino.

Os pacientes com ametropia cilíndrica de grau igual ou superior a 1,50 dioptrias (independente do eixo) em pelo menos um dos olhos, foram submetidos a exame de campimetria computadorizada. Para cada olho incluso nos critérios do estudo, foram realizados dois exames de campo visual de forma aleatória, sendo um com a utilização da correção óptica total e o outro com a utilização do equivalente esférico, em dias diferentes. O programa utilizado foi o SITA - Standard (Swedish Interactive Threshold Algorithm) 24-2, e o aparelho utilizado foi o campímetro Humphrey modelo HFA 745, Humphrey Systems (San Leandro, CA -EUA).

Na realização dos exames levou-se em consideração os seguintes aspectos para melhor execução do exame pelo paciente: ajuste adequado da distância e altura do aparelho em relação ao paciente, centralização do olho do paciente na armação de pro- va, distância mínima entre a lente e as pálpebras do paciente, colocação da lente esférica no receptáculo da armação de prova próximo ao paciente (na correção total, a lente cilíndrica foi colocada sempre no receptáculo distal ao paciente) e ajuste cuidadoso do eixo cilíndrico na correção total.

Ambos os exames de cada olho foram comparados, na tentativa de verificar se houve diferença, estatisticamente significativa, da sensibilidade aos estímulos luminosos. Para isso, foram avaliados os seguintes parâmetros do exame: "Mean Deviation” (MD), "Pattern Standard Deviation” (PSD), os índices de confiabilidade (perdas de fixação, falso-positivos e falso-negativos) e tempo de duração do exame. Foram desprezados aqueles considerados duvidosos em sua fidelidade.

Para cada parâmetro avaliado, foram calculados a média, o desvio padrão e a variância dos valores obtidos com a correção total e com o equivalente esférico, sendo então realizado teste de análise da variância (Kruskall Wallis) considerandose um valor de significância de $\mathrm{p}<0,05$.

\section{RESULTADOS}

Os resultados da comparação dos exames realizados com correção total (CT) e com equivalente esférico (EE) em relação ao "Mean Deviation" (MD), "Pattern Standard Deviation" (PSD), perda de fixação (PF), falso-positivo (FP), falso-negativo (FN) e duração do exame no que diz respeito à média, desvio padrão (DP) se encontram na tabela 1.

Dentre todas as análises apenas no critério de perda de fixação o valor de $p$ foi estatisticamente significante $(p<0,05)$.

\section{DISCUSSÃO}

Em nosso estudo, optamos pela utilização da estratégia SITA 24-2 (Swedish Interactive Threshold Algorithm) uma vez que o programa "Full Threshold" despende um longo tempo para a sua realização, o que muitas vezes dificulta a sua aplicação. A estratégia SITA foi concebida com a finalidade de diminuir a duração do exame, aumentando a quantidade de informações por unidade de tempo, oferecendo a mesma precisão na determinação de limiares em relação a "Full Threshold".

\begin{tabular}{|c|c|c|c|}
\hline \multicolumn{4}{|c|}{$\begin{array}{l}\text { Tabela 1. Média do mean deviation (MD), pattern standard deviation } \\
\text { (PSD), perda de fixação (PF), falso-positivo (FP), falso-negativo (FN) } \\
\text { e duração do exame encontrados nos exames de campimetria } \\
\text { realizados sob correção total (CT) e equivalente esférico (EE), no } \\
\text { Departamento de Oftalmologia da Santa Casa de São Paulo no perío- } \\
\text { do de dezembro de } 1999 \text { a junho de } 2000\end{array}$} \\
\hline & CT & EE & $p$ \\
\hline$M D^{*}$ & $-2,81 \pm 1,50$ & $-2,42 \pm 1,20$ & $>0,05$ \\
\hline PSD* & $1,73 \pm 0,41$ & $1,65 \pm 0,31$ & $>0,05$ \\
\hline PF & $0,10 \pm 0,08$ & $0,05 \pm 0,007$ & $<0,05$ \\
\hline $\mathrm{FP}$ & $0,01 \pm 0,02$ & $0,01 \pm 0,02$ & $>0,05$ \\
\hline $\mathrm{FN}$ & $0,02 \pm 0,03$ & $0,02 \pm 0,03$ & $>0,05$ \\
\hline TD & $5,39 \pm 1,13$ & $5,16 \pm 0,85$ & $>0,05$ \\
\hline
\end{tabular}


Um exame "Full Threshold" 24-2 dura cerca de 12 -14 minutos, enquanto o SITA Standard 24-2 dura cerca de 5 minutos $^{(2)}$.

A diferença entre as médias do "Mean Deviation”, "Pattern Standard Deviation”, falso-positivos, falso-negativos bem como da duração dos exames, realizados com a correção total e com o equivalente esférico, não foi significativa $(p>0,05)$. Portanto, não foram observadas variações, nos resultados destes parâmetros, devido ao uso do equivalente esférico ao invés da correção total.

A diferença entre as médias das perdas de fixação apresentou significância estatística ( $\mathrm{p}<0,05)$, sendo observado um menor número de perdas de fixação nos exames realizados com o uso do equivalente esférico.

Existem três motivos principais pelos quais ocorre a perda de fixação durante o exame: reflexo involuntário de fixação ${ }^{(1-2)}$ desejo de obter um bom desempenho durante o teste ${ }^{(1-2)}$; presença de alteração verdadeira que prejudique a fixação $0^{(1-2)}$.

Em nosso estudo, devido aos critérios de inclusão, pudemos concluir que as perdas de fixação não tiveram como causa, doenças oftalmológicas que levassem a impossibilidade de manter uma fixação adequada.

O campímetro utilizado em nosso estudo possui um sistema automático de detecção das perdas de fixação (emissão de estímulos na mancha cega fisiológica do paciente).

As perdas de fixação no exame de campimetria são aceitáveis até o limite de $20 \%$, acima do qual, a confiabilidade do exame é comprometida. $\mathrm{O}$ fator de aprendizado por parte do paciente pode exercer influência neste índice ${ }^{(1-2)}$.

Os exames foram realizados de forma aleatória, procurando diminuir a interferência do fator aprendizado nos resultados obtidos.

Dos 20 pacientes examinados, 14 realizaram o primeiro exame de campimetria utilizando a correção total (70\%).

Nos 6 casos que obtiveram melhora acentuada das perdas de fixação ao utilizar o equivalente esférico, 4 (66\%) já haviam realizado primeiro o exame com a correção total.

Desse modo, podemos concluir que, a ordem na qual os exames foram realizados, pode ter exercido influência nos resultados obtidos. Talvez com a realização de três exames em cada olho, ao invés de dois, o fator aprendizado fosse minimizado de modo mais adequado, uma vez que o primeiro exame serviria para a instrução do paciente, independente da correção utilizada.

A correção óptica adequada é essencial para que haja máxima sensibilidade retínica aos estímulos luminosos durante a campimetria. Uma ametropia corrigida de forma insatisfatória pode comprometer os resultados obtidos durante o exame $e^{(1-4)}$.

Diferença de apenas 1,00 dioptria na correção óptica para execução do exame pode provocar diminuição acentuada nos valores do limiar de sensibilidade ${ }^{(5)}$. Nosso trabalho não concorda com essa afirmação.

Padronizou-se que, para valores de ametropia cilíndrica até 0,75 dioptria, pode-se utilizar o equivalente esférico, e que para valores maiores do que este, deve-se optar pelo uso da correção total ${ }^{(1,4)}$.
Em nosso estudo, todos os pacientes examinados possuíam grau igual ou superior a 1,50 dioptrias cilíndricas, não sendo encontrada perda de sensibilidade aos estímulos luminosos, devido ao uso do equivalente esférico.

Nossos dados nos levaram a concluir que exames de campimetria computadorizada utilizando a estratégia SITA podem ser realizados com o equivalente esférico, dentro da média de dioptrias analisada neste estudo, sem que haja comprometimento dos resultados.

\section{CONCLUSÃO}

O estudo apresenta ressalvas, relacionadas ao tamanho da amostra e à faixa etária estudada. Referente ao tamanho da amostra, buscamos utilizar teste estatístico adequado para o número de indivíduos estudados, validando desta maneira a análise estatística. Diante da análise da validade externa pertinente à faixa etária, concluímos que os resultados obtidos só podem ser reprodutíveis em grupos que apresentem características similares ao grupo estudado.

\section{ABSTRACT}

Purpose: To compare the results in subjects undergoing visual field testing using the full cylindrical correction and the spherical equivalent. Methods: Twenty patients (35 eyes) underwent visual field testing with Humphrey (SITA 24-2) perimetry using the full cylindrical correction and the spherical equivalent at a random sequence. Mean Deviation, Standard Pattern Deviation, fixation loss, false positive and false negative results and test duration were compared. Results: No difference was found regarding Mean Deviation, Standard Pattern Deviation, false positive and false negative results and test duration. Fixation loss was significantly higher with the full cylindrical correction. Conclusion: Visual fields performed with the spherical equivalent show no change in the retinal sensitivity when compared to examinations done with the full cylindrical correction for the same eye.

Keywords: Perimetry/methods; Visual fields; Glaucoma/diagnosis; Sensorial thresholds; Refractive errors/diagnosis; Comparative study

\section{REFERÊNCIAS}

1. Anderson DR. Advanced perimetric techniques. In: Anderson DR, editor. Perimetry with and without automation, $2^{\text {nd }}$ ed. St. Louis: CV Mosby; 1987. p.448-54.

2. Costa VP. Perimetria computadorizada: um guia prático de interpretação, Rio de Janeiro: Rio Méd;2000.

3. Weinreb RN, Perlman JP. The effect of refractive correction on automated perimetric thresholds. Am J Ophthalmol. 1986;101(6):706-9.

4. American Academy of Ophthalmology. Automated perimetry. Ophthalmology. 1996;103(7):1144-51.

5. Caprioli J. Automated perimetry in glaucoma. Am J Ophthalmology. 1991; 111(2):235-9. 